\title{
16. Nationales Pathos und maskuline Attitüde: Walter Flex' Kultbuch «Der Wanderer zwischen beiden Welten»
}

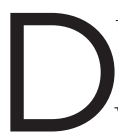

urch Vermittlung von Walther Eggert-Windegg erschien im Oktober 1916 bei C.H.Beck «Der Ein zweiter Theodor Körner. Oskar Beck

Wanderer zwischen beiden Welten» von Walter

Flex. ${ }^{1}$ Innerhalb von zehn Jahren erlebte der schmale Band 77 Auflagen. Ende Dezember 1916 waren 66oo Exemplare verkauft, im März des Folgejahrs wurde das 10000 . Exemplar gedruckt, und bis 1933 fand das Buch 340000 Käufer. Das Werk hatte Kultcharakter. Unter den Büchern, die den Ersten Weltkrieg thematisierten, war nur Erich Maria Remarques Antikriegsroman «Im Westen nichts Neues» von 1928 erfolgreicher. ${ }^{2}$ Mit über einer Million abgesetzten Exemplaren (bis 1966) gehört «Der Wanderer zwischen beiden Welten» zu den meistverkauften Büchern des Verlags C.H.Beck und zählt zu den erfolgreichsten deutschsprachigen Büchern des 2o. Jahrhunderts überhaupt. Das Buch ist nach wie vor im Buchhandel erhältlich und kann als kostenloses E-Book heruntergeladen werden.

Ein Jahrhunderterfolg, von dem sich C.H.Beck aber Mitte der 1970er Jahre trennte, als die Walter-Flex-Gedächtnisstätte in Dietzenbach zum bevorstehenden 9o. Geburts- und 6o. Todestag des Autors eine Wiederauflage des vergriffenen Werkes plante. Damals verkaufte sich das Buch bereits nicht mehr gut, und so sprachen auch kaufmännische Erwägungen gegen eine Neuauflage. Auch eine Dokumentation aus dem Nachlass von Walter Flex wollte man in München nicht verlegen. Man trat stattdessen die Verlagsrechte ohne Aufsehen an den nationalkonservativen OrionHeimreiter-Verlag ab. ${ }^{5}$ Für Wolfgang Beck, der seit 1973 die verlegerische Verantwortung für das geistes- und kulturwissenschaftliche Programm trug, dürfte offenkundig gewesen sein, dass weder das Buch noch seine Leserschaft dem liberalen Bild des Verlags entsprachen, das er zu vermitteln sich vorgenommen hatte. Wolfgang Beck hatte damit das Anathem über einen Titel verhängt, der exemplarisch für nicht wenige nationalistische und kriegsverherrlichende Bücher steht, die der Verlag nach dem Ausbruch des Ersten Weltkriegs veröffentlichte.

«Der Wanderer zwischen beiden Welten» war «das lang wirkende 
Kriegs- und Generationsbuch idealistischer Jugendlichkeit», ${ }^{4}$ das C.H.Beck bei abertausend Jugendbewegten und Kriegsbegeisterten bekannt machte. Sein Autor Walter Flex war 1887 geboren worden, stammte aus dem thüringischen Bildungsbürgertum, hatte in Erlangen und Straßburg Geschichte und Germanistik studiert und war mit einer literaturgeschichtlichen Arbeit promoviert worden. Der begeisterte Burschenschafter veröffentlichte bereits als Student seine ersten literarischen Versuche und träumte von einem Leben als freier Schriftsteller. Um ein Auskommen zu finden, musste er jedoch als Hauslehrer arbeiten. Zeitweise unterrichtete er Klaus von Bismarck, den Enkel des Eisernen Kanzlers, im hinterpommerschen Varzin. Nachdem er 1912 nach Friedrichsruh übersiedelt war, um dort zwei weitere Bismarckenkel zu unterrichten und das Familienarchiv zu ordnen, überwarf er sich jedoch mit den Bismarcks, die seinen patriotischen Phantasien nicht entsprachen und bei Tisch Englisch parlierten. Während seines Aufenthalts in Friedrichsruh brachte er verschiedene Bismarckwerke zu Papier, darunter ein Trauerspiel «Klaus von Bismarck - Eine Kanzlertragödie». Im August 1914 meldete sich Walter Flex wie so viele seiner Altersgenossen als Kriegsfreiwilliger und zog begeistert ins Feld. An der Front schrieb er kriegsverherrlichende Gedichte, die er an die «Tägliche Rundschau» und an «Westermanns Monatshefte» sandte. Noch 1914, erschien seine Lyrik in der Anthologie «Ein Volk in Eisen», die rasch neu aufgelegt und zu einem «Ehrenmal» für seinen «für Kaiser und Reich gefallenen» jüngeren Bruder Otto wurde. ${ }^{5}$ Kriegsdichtung war das literarische Genus der Stunde. Folgt man einer zeitgenössischen Schätzung, so wurden anderthalb Millionen Gedichte allein im Monat August verfasst und den Redaktionen zugesandt. ${ }^{6}$

Es waren die Gedichte, die C.H.Beck auf den jungen Schriftsteller aufmerksam werden ließen. An dem kollektiven Lyrikrausch wollte auch der Münchner Verlag partizipieren. Der Verlagsredakteur Walther EggertWindegg fragte Ende September 1914, mehrere Autoren, darunter Ferdinand Avenarius, Hans Bethge, Michael Georg Conrad, Richard Dehmel, Otto Ernst, Gerhart Hauptmann und Hermann Hesse, ob sie bereit wären, Gedichte, die sie bei Kriegsbeginn in der Presse veröffentlicht hatten, für eine «kleine Auswahl» zur Verfügung zu stellen, «die zur Erhaltung und Steigerung der vaterländischen Stimmung in Volk und Heer beitragen, aber auch als ein literarisches Denkmal aus dieser großen Zeit übrig bleiben soll». Den Reingewinn wollte man zur Unterstützung notleidender Berufsgenossen an die Ortsgruppe München des Schutzverbands deutscher Schriftsteller überweisen. ${ }^{7}$ Eggert-Windegg bat damals auch Walter Flex, 
seine Gedichte «Zukunftsspruch» und «Preußischer Fahneneid» zur Veröffentlichung freizugeben. Mit seinen Versen feierte dieser «tausend Brüder», die Deutschlands Zukunft, an der sie «blutig» bauten, nie mit Augen sähen, pries den Eid auf den König von Preußen als Symbol für soldatische Treue und ließ den Einzelnen in der Gemeinschaft aufgehen: «Wer auf die preußische Fahne schwört, / Hat nichts mehr, was ihm selber gehört.» ${ }^{8}$ Die Sammlung der Gedichte gab Eggert-Windegg selbst unter dem Titel «Der Deutsche Krieg in ausgewählten Dichtungen» Ende 1914 heraus; für zwei Mark fünfzig erhielt der Leser, schenkt man dem «Reichsboten» Glauben, «eines der schönsten und preiswertesten Bücher» des ersten Kriegsjahrs. Der Band erschien 1917 in dritter Auflage. ${ }^{9}$

Walter Flex verbrachte die ersten Kriegsmonate im Stellungskrieg in Lothringen. Dort entstand am Totensonntag das Gedicht «Vom großen Abendmahl», in dem eine martialische Lesart der Transsubstantiation vorgetragen wurde: «Zum Altar ward das Feld der Völkerschlacht. / Aus deutschem Blut ist Christi Wein bereitet, / und in dem Blut der Reinsten wirkt die Macht / des Herrn, der durch die heil'ge Wandlung schreitet.» Für die Weihnachtsfeier seines Infanterieregiments schrieb Flex das «Weihnachtsmärchen von den toten Soldaten», das er am Heiligen Abend 1914, bei der Christfeier seiner fünften Kompanie «vor dem tannenbekränzten Altar der Dorfkirche» vorlas. Die «schweren Geschütze dröhnten dazu eine nachdenkliche Begleitung». ${ }^{10}$ Die Texte erklärten den blutigen Grabenkrieg zum Leidensweg des deutschen Volkes und verklärten den Tod des einzelnen Soldaten zum Martyrium in der Nachfolge Christi. C.H.Beck veröffentlichte im zweiten Kriegsjahr die Stücke zusammen mit einem Feldpostbrief, der «Nachtgedanken» enthielt, unter dem Titel «Vom großen Abendmahl. Verse und Gedanken aus dem Feld». Das Büchlein gehöre «wohl zu dem Schönsten, was aus dem inneren Erlebnis des Krieges über die sittliche Idee des Heldentodes geschrieben worden ist. Es ist namentlich ein wahres Trostbüchlein für alle, die einen toten Soldaten haben.» ${ }^{11}$ Die 10. Auflage wurde 1918 herausgegeben, die 39. im Jahr 1920; 1927 waren über 100 ooo Exemplare verkauft und 1944 weit über 160 ooo. ${ }^{12}$

Die Begeisterung für die Kriegsgedichte machte Flex zum bekannten Poeten, und C.H.Beck wurde zu einem der wichtigsten Verlage der geistigen Mobilmachung. Das Verlagsprogramm atmete den «Geist von 1914».13 Eggert-Windegg schob 1915 unter dem Titel «Der Barde» eine Sammlung nach, die die deutsche Geschichte von ihren Anfängen bis zur Gegenwart in Gedichten zu erfassen suchte; eine dritte Auflage erschien 1935. Will Vesper erlebte seinen poetischen Durchbruch mit den Gedichten «Vom 


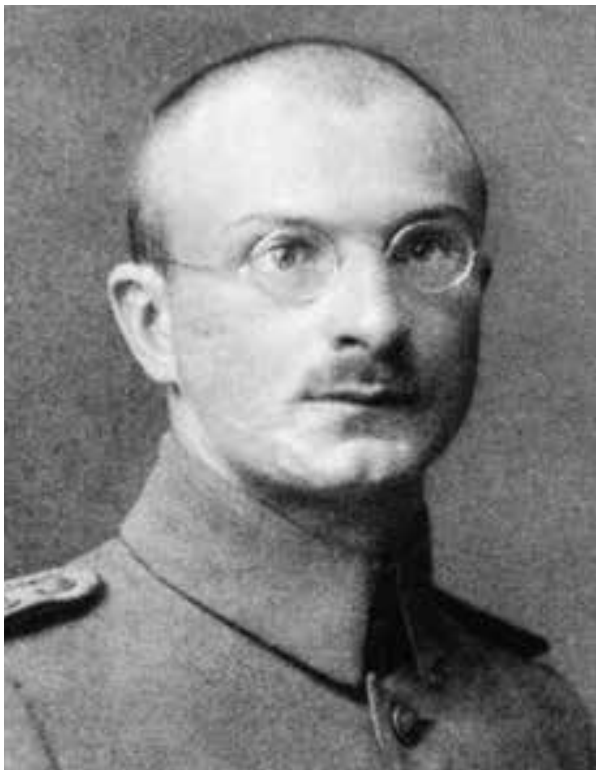

Walter Flex

großen Krieg» (1914) und «Der deutschen Seele Trost» (1915). Hans Benzmann veröffentlichte 1915 Kriegsgedichte «Für Kaiser und Reich». Doch auch andere Texte wurden in das Programm aufgenommen. Der Arbeiterpfarrer Johann Gottlieb Cordes verfasste «Briefe in die Front» (1914), die für seine ehemaligen Konfirmanden gedacht waren. In «handlichem Format» war das Büchlein ein «willkommener Lesestoff» für die «Feldgrauen». Der Erlanger Kirchenhistoriker Christian Bürckstümmer brachte Ende 1914, eine Sammlung von Kriegspredigten heraus, unter dem treffenden Titel «Eine feste Burg ist unser Gott». An der nationalistischen Begeisterung beteiligte man sich auch durch Hetzschriften, die gegen Deutschlands Feinde gerichtet waren. Der Schriftsteller Karl Strecker etwa bot in seinem Buch «England im Spiegel der Kulturmenschheit» (1914) eine «wohlgeordnete Sammlung von Aussprüchen hervorragender Männer aller Zeiten und Völker über das perfide Albion». Für zwei Mark konnte man sich bestätigen lassen, dass der deutsche Kampf gerecht sei und Deutschland «die Sache der Menschheit» führe. ${ }^{14}$ Hinzu kamen Johannes Müllers «Reden über den Krieg» (1914/15), die den Krieg «als Schicksal und Erlebnis», «als Not und Aufschwung», «als Gericht und Aufgabe», ferner den «Tod fürs Vaterland und die Hinterbliebenen» und den «Krieg als religiöses Erlebnis» behandelten. Die «Chronik des Deutschen Krieges nach amtlichen Berichten und zeitgenössischen Kundgebungen» erschien erstmals 1914; 1917 lag bereits der zehnte Band vor, der das Geschehen vom 21. Oktober bis 30. November 1915 auf über 500 Seiten darstellte.

Der bürgerliche Traditionsverlag publizierte in rascher Folge patriotische, chauvinistische und kriegsverherrlichende Texte, die mit nationalem Pathos Gott und Vaterland priesen, den Tod auf dem Schlachtfeld als höchste Erfüllung männlichen Daseins glorifizierten, den geborenen Führer verherrlichten, Kameradschaft, Treue und Aufopferung verlangten und bedingungslosen Gehorsam einforderten. Kritik an der Vorkriegszeit fand sich nicht, man lebte und handelte im Hier und Jetzt, zum größeren Lobe des dreieinigen Gottes und des Kaisers. Als der Sieg dann in weite 
Ferne rückte, sollten neue Titel und Nachdrucke den Willen zum Durchhalten stärken.

Die Schlacht um Verdun tobte, als Walter Flex' autobiographischer Bestseller «Der Wanderer zwischen beiden Welten. Ein Kriegserlebnis» erschien. Am Anfang des dünnen Bandes findet sich das berühmte Gedicht «Wildgänse rauschen durch die Nacht», das sich bald aus seinem ursprünglichen Kontext löste und eine eigene Rezeptionsgeschichte entwickelte. Bis in die 1970er Jahre wurde das Lied in linken und rechten Kreisen gesungen: «Wildgänse rauschen durch die Nacht / Mit schrillem Schrei nach Norden - / Unstäte Fahrt! Habt acht, habt acht! / Die Welt ist voller Morden. // Fahrt durch die nachtdurchwogte Welt, / Graureisige Geschwader! / Fahlhelle zuckt, und Schlachtruf gellt, / Weit wallt und wogt der Hader. // Rausch' zu, fahr' zu, du graues Heer! / Rauscht zu, fahrt zu nach Norden! / Fahrt ihr nach Süden übers Meer - / Was ist aus uns geworden! // Wir sind wie ihr ein graues Heer / Und fahr'n in Kaisers Namen, / Und fahr'n wir ohne Wiederkehr, / Rauscht uns im Herbst ein Amen!»15

Im Mittelpunkt des «Wanderers» steht der liberale, jugendbewegte Theologiestudent Ernst Wurche, der sich wie der sieben Jahre ältere Walter Flex freiwillig zum Kriegsdienst meldet und in dessen Tornister Goethe, Nietzsche und die Feldausgabe des Neuen Testaments zusammenfinden. Beide lernen sich im März 1915 an der Westfront kennen und absolvieren anschließend gemeinsam einen Offizierslehrgang. Die Fahrt an die Ostfront macht aus den beiden jungen Soldaten Freunde. Flex singt darin mitten in den Grabenkämpfen das Hohelied auf die Freundschaft der jungen Frontoffiziere. Er erkennt in Wurche das körperliche und geistige Jünglingsideal des Wandervogels. Homoerotischer Körperkult und intensives Naturerlebnis verschmelzen, und der Krieg wird zur Idylle stärkster persönlicher Gefühle. Der Kampf reißt die beiden Freunde, die den Sturm auf die russischen Stellungen überleben, schließlich auseinander. Wenig später, am 23. August 1915, erfährt Flex von Wurches Tod. Das Buch endet mit einem Zwiegespräch zwischen Flex und dem getöteten Freund, der Trost spendet und den Trauernden aufrichtet. Im Krieg entsteht eine neue Volksgemeinschaft, die im Totengedenken zueinander findet. Der Heldentod wird zur imitatio Christi, die dem Gefallenen Unsterblichkeit und der Nation Erlösung verleiht. Die Erzählung endet im Frühjahr 1916 und schließt mit dem Wort aus dem Weihnachtsevangelium: «Fürchtet euch nicht!» ${ }^{16}$

Das Buch wurde in den Medien gefeiert. Begeisterte Besprechungen erschienen in der «Ostdeutschen Rundschau», der «Monatsschrift für höhere Schulen» und dem «Wandervogel». Die Nachfrage war gewaltig, 1500 Ex- 
emplare des Neudrucks wurden im Februar 1917 dem Buchbinder übergeben, und im Juli $1917 \mathrm{kam}$ bereits die vierte Auflage heraus. Dennoch entschied sich der Verlag gegen eine wohlfeile Feldausgabe, da auch ein leichterer Umschlag nicht geholfen hätte, den Preis so weit zu senken, dass das Bändchen für zwei Mark hätte geliefert werden können. Es blieb bei der Ausgabe für 2,50 Mark. Zugleich nutzte der Verlag die Gunst der Stunde und brachte eine Neuauflage «Vom großen Abendmahl» heraus. Mit seinem Namen ließen sich gute Geschäfte machen. ${ }^{17}$ Die Papierknappheit, die sich immer stärker bemerkbar machte und den Druck manches wissenschaftlichen Verlagsprojekts beeinträchtigte, verhinderte nicht die Verbreitung dieser Literatur. Statt auf «blütenweißem Friedenspapier» wurde jetzt auf «graufarbenem Kriegspapier» gedruckt. ${ }^{18}$

Die Kommunikation mit dem Verlag war dicht, obwohl der Autor im Feld stand. Flex war über Nacht mit Hilfe von C.H.Beck zu einer Berühmtheit geworden. An ihn schrieben sowohl der Verleger, Oskar Beck, als auch der Verlagsmitarbeiter Walther Eggert-Windegg. Der eine war für Honorarfragen zuständig, der andere betreute neue Projekte. In dem Briefwechsel mit Walter Flex zeigt sich beispielhaft die Differenzierung der Arbeitswelt im Verlag. Eggert-Windegg war de facto der erste Lektor des Verlags C.H.Beck, auch wenn er noch nicht als solcher bezeichnet wurde.

Am 5. Juni 1917 besprach Oskar Beck mit Flex einen neuen Abrechnungsmodus. Hintergrund war, dass die Herstellungskosten durch «die stetig steigenden Arbeitslöhne sowie die fortwährende Erhöhung der Preise aller Materialien» erheblich gestiegen waren. Vor allem das Papier war um 150 Prozent gegenüber dem Preis in Friedenszeiten teurer geworden. Da man im Juli 1916 vereinbart hatte, die Hälfte des Reingewinns nach Abzug aller Kosten dem Autor zuzugestehen, reduzierte sich der Gewinnanteil für Flex ständig. Dennoch verdiente der junge Kriegspoet gut, wie die erhaltenen Honorarabrechnungen belegen. Für die zweite, dritte und vierte Auflage des «Wanderers» sowie die dritte und vierte Auflage des «Abendmahls» erhielt Flex allein in der ersten Hälfte des Jahres 1917 ein Gesamthonorar von 3938,86 Mark. ${ }^{19}$ Der Verleger bot dem auflagenstarken Autor jedoch an, ein festes Honorar zu vereinbaren, und zwar beim «Wanderer» 750 Mark für jede Auflage von 3300 Exemplaren und beim «Abendmahl» 200 Mark für jede Auflage von 2200 Exemplaren. Flex zeigte sich mit der für ihn günstigen Vertragsänderung einverstanden. ${ }^{20}$ Am 11. August 1917 wurde Flex für die beiden Bände ein Honorar von 1150 Mark überwiesen. ${ }^{21}$ 
Bei dem geplanten Gedichtband «Im Felde zwischen Nacht und Tag» verhielt sich die Sache anders. Oskar Beck bestand in dem Fall auf Gewinnteilung, «da sich über das Schicksal eines Gedichtbandes, und wäre er noch so trefflich, schwer etwas Bestimmtes voraussagen lässt». Bessere Chancen räumte er einem Novellenband mit Geschichten aus dem Dreißigjährigen Krieg ein, der zu Weihnachten herauskommen sollte. Der Verleger schloss seinen Brief mit einer Reflexion über das Verhältnis von literarischem Markt und Weltkrieg. Gewiss, er schrieb nationalistische Klischees und Stereotype fort, aber von der Euphorie des August 1914, war im Sommer 1917 nichts mehr übrig geblieben: «Die Erfolge von «Wanderer〉 und «Abendmahl> sind auch um deswillen so erfreulich, weil darin der Beweis liegt, dass der ideale Sinn trotz der langen, zermürbenden Kriegsdauer doch in Volk und Heer nicht erloschen ist. Wenn wir nur bald einem guten Frieden entgegensehen könnten! Es wird freilich, auch wenn die sonstige Voraussetzung, nämlich die Bereitwilligkeit unserer Gegner, ihn abzuschließen, vorhanden ist, für unsere deutsche Diplomatie ein schweres Stück Arbeit werden. Wenn man in den maßgebenden Kreisen sich nur über die Kriegsziele und ihre Tragweite recht klar wäre! An Schlauheit sind die Staatsmänner der Feinde den unsrigen ja leider auch sehr überlegen!»²

Am 2o.September 1917 war der geplante Novellenband auch Gegenstand eines langen Briefes von Eggert-Windegg an Flex, der erkennen lässt, wie der Redakteur als Lektor seinen Autor beriet, warnte und ermunterte. Eggert-Windegg ließ keinen Zweifel daran, dass er in Übereinstimmung mit dem Verleger schrieb. Aus den Novellen, die zuvor bereits einzeln in Zeitschriften erschienen waren, könne man sicher «ein einheitliches, feines Büchlein» machen, das ein größerer Erfolg würde. Dennoch riet er von einer Veröffentlichung ab, da ihm die bereits publizierten Geschichten zum Dreißigjährigen Krieg nicht attraktiv erschienen. Nicht die Vergangenheit, sondern die Gegenwart sollte im Herbst 1917 literarisch abgebildet werden. Vorsichtig versuchte Eggert-Windegg, dem jungen Schriftsteller seine Einschätzung zu vermitteln. Er habe in erster Linie geprüft, ließ er sich vernehmen, ob durch eine Publikation auch Flex’ «subtilsten persönlichen Interessen gefördert würden», «ob die seit dem 〈Abendmahl> und dem «Wanderer〉 hochansteigende Linie» seiner literarischen Karriere «weiter emporgeführt und nicht etwa, auch nur im geringsten, abgebogen würde». Um in dieser heiklen Frage Rat geben zu können, bedurfte es eines vertrauensvollen Verhältnisses zwischen Autor und Lektor, das emphatisch erneuert und bestätigt werden musste. Eggert-Windegg sah sich zu «besonderen Erwägungen und Rücksichten 
verpflichtet» und schrieb: «Sie selbst, sehr verehrter Herr, haben Ihre Bedenken gehabt und mir auch angedeutet und haben darum besondere Strenge des Urteils von mir verlangt. Es ist nun wohl kaum nötig oder auch nur angebracht, dass ich mich im Einzelnen über die Stücke auslasse, sondern es genügt wohl die Feststellung des Gesamteindruckes, fast möchte ich sagen, des Gemeingefühls, die mir - immer im Hinblick auf die Gegenwart Ihres Schaffens - verblieben sind: ich hätte die Veröffentlichung der gesammelten (ich betone das Wort) Stücke und Studien eben jetzt als einen Rückblick empfunden, zu dem noch keine passende Zeit und Gelegenheit wäre, da Ihr Schaffen spürbar vorwärts drängt mit einem stürmenden Pulse und einem sittlichen Pathos, die in den Novellen oder Erzählungen und Studien nicht so vorhanden sind, noch zu sein brauchten und sein konnten. Auf andere Art ausgedrückt: die Novellen hätten ‘iterarisch> anmuten und das Bild Ihres gegenwärtigen, ganz von dem lebendigsten Erleben der unerhörten Gegenwart und von leidenschaftlicher (unbewusster) Mission erfüllten Schaffens und Bedeutens stören können. Ich weiß nicht, ob ich mich Ihnen damit in diesen kaum wägbaren und sagbaren Dingen habe verständlich machen können, und wünschte doch sehr, von Ihnen verstanden zu werden. Brauche ich noch hinzuzufügen, dass mit all dem gegen den Wert der Novellen an sich nichts gesagt ist und wir später, ich hoffe es, wohl darauf zurückkommen können.» Stattdessen lobte er die Novelle «Wolf Eschenlohr», an der Flex im Feld arbeitete, überschwenglich, da «der gute Geist dieses neuen Buches» ihm «schon auf den ersten Seiten» entgegengeweht sei. «Die Gestalten stehen schon jetzt in Lebensfülle und vertraut vor mir; ich brenne darauf, ihre Schicksale, für die sie so rasch und so ganz die Teilnahme des Lesers erweckt haben, mitzuerleben: senden Sie wenigstens Stück um Stück! Es wird alles wohl verwahrt werden. «Wolf Eschenlohr〉 wird, das sehe und sage ich schon jetzt, ein gutes und schönes Buch sein.» ${ }^{23}$

Walther Eggert-Windegg, im Verlag C.H.Beck Lektor avant la lettre, gestaltete aktiv das Verlagsprogramm. Der Autor vertraute ihm. Den Novellenband stellte er zurück, um stattdessen an «Wolf Eschenlohr» zu schreiben, in dem unter Rückgriff auf antisemitische Vorurteile die Hauptfigur Wolf Eschenlohr von seinem ehemaligen jüdischen Schulkameraden Moritz Hirschberg abgegrenzt wird. Beide melden sich freiwillig zum Kriegsdienst, doch nur der deutschstämmige Protagonist erkennt die Größe des historischen Moments, während Hirschberg die Euphorie nicht versteht und sie rational «zergliedert»: Hier der um Assimilation bemühte Jude mit «spöttischer Fistelstimme» und «verschwitzten Gläsern» eines «schiefsitzenden 
Goldzwickers», selbst seine «Affenähnlichkeit» beklagend, dort der «schlanke Junge» mit «hellem Gesicht», ausgezeichnet durch «körperliche Zucht und Gewandtheit». ${ }^{24}$ In der Novelle «Wolf Eschenlohr» vollzog Flex die rassistische Ausgrenzung der artfremden Juden aus der deutschen Volksgemeinschaft, und Eggert-Windegg applaudierte der antisemitischen Agitation, die in einer nationalistisch gesinnten Publizistik weite Verbreitung gefunden hatte, als der schnelle Sieg sich nicht einstellen wollte.

Keine vier Wochen später war Walter Flex tot. Bei der Erstürmung der estnischen Insel Ösel (heute Saaremaa) wurde er am 15. Oktober 1917 schwer verwundet. Die Überlieferung will, dass er mit gezücktem Säbel auf einem erbeuteten Pferd gegen den russischen Feind ritt. Das zweite Kapitel von «Wolf Eschenlohr», das er in der Kartentasche bei sich trug, soll von der tödlichen Kugel durchlöchert worden sein. Am nächsten Tag erlag er im Lazarett seinen Verletzungen. Sein Leichnam wurde auf Ösel beigesetzt. ${ }^{25}$ Sobald die Nachricht nach München gelangt war, schrieb Oskar Beck an Rudolf Flex, den in Eisenach lebenden Vater des Gefallenen, einen ausführlichen Kondolenzbrief, darin er den «Heldentod» beschwor und eine Märtyrerlegende schuf, die den Erfolg der Bücher auch in Zukunft sicherte. Es sei ihm leider nicht vergönnt gewesen, «den trefflichen Verfasser des ‘Wanderers zwischen beiden Welten` von Angesicht zu Angesicht kennenzulernen». Gleichwohl habe er ihn «durch dieses seelenvolle Buch von Herzen liebgewonnen». Walter Flex sei nun einen Tod gestorben, «wie er ihn sich selbst wohl nicht schöner wünschen mochte. An der Spitze seiner Kompanie, bei einer Waffentat, die zu den schönsten dieses von rühmlichen Waffentaten überreichen Krieges zählt, hat er die Todeswunde erhalten. Als ein zweiter Theodor Körner wird er fortleben im deutschen Volke und in der deutschen Literaturgeschichte.» Durch seine Bücher habe der Verstorbene einen großen Kreis von Freunden gewonnen. Tausende betrauerten den so früh Gestorbenen. «Wie groß aber mag erst die Trauer der Eltern sein um einen solchen Sohn! Er selbst mahnte ja: Totenklage ist ein arger Dienst. Heimatrecht verlangt er für unsere Toten. So scheint es nicht in seinem Sinn, wenn wir um ihn klagen. Und doch füllen sich unsere Augen mit Tränen, wenn wir daran denken, was der vom höchsten Idealismus erfüllte junge Dichter, der erst seine Schwingen recht zu entfalten im Begriffe war, seinem deutschen Volke noch hätte vererben können, wenn ihm ein längeres Leben vergönnt gewesen wäre. Einen Dichter von Gottes Gnaden und von solcher Gesinnung, wie Walter Flex einer war, haben wir so notwendig gebraucht! Nun müssen wir die wenigen Werke, die er uns geschenkt hat, umso höher 
schätzen. Sie sind von so ausgeprägter Eigenart, dass sie uns immerhin ein scharf umrissenes Bild von ihrem Schöpfer zu gewinnen gestatten.» Der Verleger erlaubte am Ende einen Einblick in seine Seele. Es quälte ihn die Angst um seinen einzigen Sohn Heinrich, der seit Kriegsbeginn an der Front stand und einmal leicht verwundet worden war. Er schloss mit den Worten: «Der gnädige Gott hat sein Leben aber bis jetzt begleitet. Doch ist uns die tägliche Sorge um das teure Leben eines lieben Kindes nur zu vertraut.» ${ }^{26}$

Immerhin war Oskar Beck Geschäftsmann genug, um auch in dieser Situation an seinen Verlag zu denken. Befriedigt nahm er zur Kenntnis, dass der letzte Gedichtband «Im Felde zwischen Nacht und Tag» gut lief. Binnen Jahresfrist sollte er 21 Auflagen erleben. Weitblickend entwarf er in diesem Kondolenzschreiben ein Editionsprogramm für Walter Flex. Die Sammlung kleiner Novellen, die schon in München lag, von deren Veröffentlichung Eggert-Windegg allerdings abgeraten hatte, erschien jetzt verlegerisch doch interessant, da sie der Verfasser selbst zusammengestellt hatte. Also wollte man das Bändchen rasch der Öffentlichkeit übergeben. Die Texte erschienen unter dem Titel «Wallensteins Antlitz» im Jahr 1918. Außerdem besaß der Verlag den «hoffnungsvollen Anfang» der größeren Novelle «Wolf Eschenlohr», denn Walter Flex hatte das «schön mit eigener Hand ins Reine geschriebene Manuskript» nach München geschickt. Oskar Beck hoffte, dass es gelänge, «aus Aufzeichnungen und Entwürfen, die sich hoffentlich im Gepäck des Verewigten finden, diese Schöpfung einigermaßen zu ergänzen und zu runden». ${ }^{27}$ Eggert-Windegg bot seine Hilfe an. Das antisemitische Buch erschien 1919 in erster Auflage mit einem Vorwort des Bruders Konrad Flex. 1943 waren über 100000 Exemplare verkauft. Schließlich dachte man daran, eine Auswahl aus den Briefen zu veranstalten. Sie kam 1927 in die Buchhandlungen. Zwei Jahre zuvor hatte C.H.Beck bereits in zwei Bänden die «Gesammelten Werke» von Walter Flex verlegt.

Nach der Niederlage im Ersten Weltkrieg zog der Verlag C.H.Beck Gewinn aus dem Werk eines Autors, der zum Künder jenes «unbeugsamen und $\mathrm{zu}$ keiner Konzession bereiten Idealismus» wurde, «der in allem Grauen des Krieges, im Tode und selbst im Gedanken an den Untergang des eigenen Volkes den Ewigkeitsglauben an Gotteskindschaft und Menschenbruderschaft» festgehalten habe. «Im Kampf um die gerechte Sache» müsse «ein Volk auch den eigenen Tod erleiden können, ohne an der sittlichen Weltordnung irre zu werden.» ${ }^{28}$ Walter Flex war in der Weimarer Republik angekommen. Dem sinnlosen Opfer sollte Sinn verliehen wer- 
den. Gerade sein «Wanderer» wurde zum Katechismus der Generation von Langemarck. Gewiss, der liberale Traditionsverlag wurde nicht zum rechten Weltanschauungsverlag, aber er wechselte mit den auflagenstarken Schriften des jugendbewegten Dichters in das konservative Milieu, dessen Repräsentanten die erste deutsche Demokratie ablehnten, sich in die vermeintlich heile Welt des Kaiserreichs zurücksehnten, antisemitische Vorurteile verbreiteten und die Erneuerung des deutschen Volkes herbeiwünschten. Mit Oswald Spengler hatte nach 1918 zudem ein Theoretiker der Konservativen Revolution zu C.H.Beck gefunden, der dem weltanschaulichen Programmbereich auch intellektuelle Ausstrahlung verlieh.

Walter Flex war im kollektiven Gedächtnis der Weimarer Republik fest verankert. Der Mythos seines Heldentums nährte nicht nur in der bündischen Jugend eine antimoderne Protesthaltung. Sein Grab auf der Insel Ösel wurde zur Wallfahrtsstätte. 1925 wurde in Eisenach die Walter-FlexGedächtnisstiftung gegründet, und die «Wildgänse» flogen in die deutschen Lieder- und Schulbücher. Die letzte Strophe wurde angepasst: Jetzt fuhr das graue Heer nicht mehr in Kaisers, sondern in Deutschlands Namen. Das von C.H.Beck verlegte Euvre bot nach 1933 «mit seinem nationalistischen Totenkult, seiner Volksgemeinschaftsmythologie, seiner latenten Kriegsbereitschaft und seiner Adoration des charismatischen Führers» hinreichend «Ansatzpunkte, um ihm Anerkennung und Gefolgschaft» bei den Nationalsozialisten zu verschaffen. ${ }^{29}$ Auch wenn einige Nazi-Funktionäre Vorbehalte gegen den angeblichen «Judenstämmling» Flex hatten und Anstoß an der Omnipräsenz christlicher Zitate und Motive nahmen, galt er zwischen 1933 und 1945 als Klassiker der deutschen Literatur, den man gelesen haben musste. «Der Wanderer zwischen beiden Welten» war Pflichtlektüre in den Schulen. Das «Wildgänse»-Lied, inzwischen schneidig vertont, wurde in der Hitlerjugend, bei Soldatenaufmärschen, im Reichsarbeitsdienst, in der Wehrmacht, der SA und der Waffen-SS gesungen. Walter Flex hatte, wie es in einer zeitgenössischen Literaturgeschichte hieß, den «Opfertod als Vollendung von Leben und Werk» erlitten. ${ }^{30}$ C.H.Beck verdiente gut an dem früh Vollendeten. 1937 erschien pünktlich zum 2o. Todes- und 50. Geburtstag eine Gedächtnisausgabe in Sonderausstattung, und 1939 gab man eine Auswahl aus den Kriegsbriefen heraus - unter dem Titel: «Für dich, mein Vaterland!»

1944 erschien «Der Wanderer zwischen beiden Welten» als «Frontbuchhandelsausgabe» für die Deutsche Wehrmacht. ${ }^{31}$ Der Wanderer zwischen Leben und Tod war der Begleiter im «totalen Krieg». Als der «Großdeutsche Rundfunk» am Abend des 1. Mai 1945 meldete, dass der «Führer» in 
der Reichshauptstadt «bis zum letzten Atemzuge gegen den Bolschewismus kämpfend» gefallen sei, ertönten nicht nur Trauermärsche, sondern eine Stimme verlas den Abschnitt aus dem «Wanderer», in dem der Tod Ernst Wurches beschrieben wurde. Aus dem «Requiem für Leutnant Wurche» war ein «Requiem für Adolf Hitler» geworden. ${ }^{52}$ 